\title{
Unified force law for granular impact cratering
}

\author{
HIROAKI KATSURAGI* AND DOUGLAS J. DURIAN ${ }^{\dagger}$ \\ Department of Physics and Astronomy, University of Pennsylvania, Philadelphia, Pennsylvania 19104-6396, USA \\ *Permanent address: Department of Applied Science for Electronics and Materials, Kyushu University, Kasuga, Fukuoka 816-8580, Japan \\ †e-mail: djdurian@physics.upenn.edu
}

Experiments on the low-speed impact of solid objects into granular media have been used both to mimic geophysical events $^{1-5}$ and to probe the unusual nature of the granular state of matter ${ }^{6-10}$. Observations have been interpreted in terms of conflicting stopping forces: product of powers of projectile depth and speed ${ }^{6}$; linear in speed ${ }^{7}$; constant, proportional to the initial impact speed $^{8}$; and proportional to depth ${ }^{9,10}$. This is reminiscent of high-speed ballistics impact in the nineteenth and twentieth centuries, when a plethora of empirical rules were proposed ${ }^{11,12}$. To make progress, we developed a means to measure projectile dynamics with $100 \mathrm{~nm}$ and $20 \mu$ s precision. For a 1-inch-diameter steel sphere dropped from a wide range of heights into noncohesive glass beads, we reproduce previous observations ${ }^{6-10}$ either as reasonable approximations or as limiting behaviours. Furthermore, we demonstrate that the interaction between the projectile and the medium can be decomposed into the sum of velocity-dependent inertial drag plus depth-dependent friction. Thus, we achieve a unified description of low-speed impact phenomena and show that the complex response of granular materials to impact, although fundamentally different from that of liquids and solids, can be simply understood.

To measure dynamics, we use a line-scan digital CCD (chargecoupled device) camera to image a finely striped transparent rod attached vertically to the top of the projectile (see the Methods section). The instantaneous speed is the key quantity, deduced from the displacement of the striped pattern between successive frames. The temporal precision is $20 \mu \mathrm{s}$, set by the $50 \mathrm{kHz}$ frame rate of the line-scan camera. The position resolution is $100 \mathrm{~nm}$, set by the $3.8 \mu \mathrm{m}$ per pixel magnification divided by the square root of the number of pixels. These combine to give a velocity resolution of $0.5 \mathrm{~cm} \mathrm{~s}^{-1}$. Besides measurement fidelity, another advantage of our method is that it applies even to very deep impacts - as long as the striped rod does not submerge.

Our complete dynamics data set is shown in Fig. 1, which shows position $z$, velocity $v$, and acceleration $a$, versus time $t$, for initial impact speeds, $v_{0}$, ranging from 0 to $-400 \mathrm{~cm} \mathrm{~s}^{-1}$. Time is measured from initial impact; position is measured upwards from the granular surface, opposite to gravity. A striking feature is that, although the final position is approached smoothly, the velocity vanishes abruptly with a discontinuity in acceleration. Similar behaviour is evident in data from an embedded accelerometer (P. B. Umbanhowar, private communication, 2004; J. C. Amato, private communication, 2005). This is counter to the viscous approach to a stable equilibrium, where acceleration vanishes continuously, but it permits the stopping time, $t_{\text {stop, to be }}$ easily gauged from the velocity versus time data. Note that $t_{\text {stop }}$ actually decreases with increasing impact speed; surprisingly, deeper penetration requires less time. Evidently, granular matter is very different from ordinary solids and liquids in its resistance to penetration.

We begin the analysis by comparing our impact data with the seemingly contradictory trends reported in refs 6-10. First, the stop time is plotted versus $v_{0}$ in the inset of Fig. 1b. As in ref. 8, for a 4.46-cm-diameter steel cylinder dropped sideways onto a bed of $0.46-0.64 \mathrm{~cm}$ diameter rods, the stopping time, $t_{\text {stop }}$, seems constant for fast impacts; however, this is only the limiting behaviour as $t_{\text {stop }}$ increases for slower impacts. This trend for stopping time with impact speed may also be seen in the raw data of ref. 10. Second, the instantaneous acceleration is plotted versus depth in the inset of Fig. $1 \mathrm{c}$ for the case $v_{0}=0$. As in ref. 9 , for 4 -cm-diameter spheres of varying mass released from rest at the surface of a bed of $40 \mu \mathrm{m}$ sand grains, $a$ seems equal to $-g$ plus a Coulomb friction term proportional to depth; however, this is only the limiting behaviour as we find in the main plot that the acceleration at $z=0$ increases with impact speed. Third, the absolute final penetration depth, $d$, is plotted in Fig. 2 versus both the total drop distance, $H=h+d$, and versus the impact speed, $v_{0}=-(2 g h)^{1 / 2}$, where $h$ is the free-fall distance and $g=980 \mathrm{~cm} \mathrm{~s}^{-2}$. As in refs 6,13 , for a wide variety of projectiles and grains, $d$ is well approximated by $\left(d_{0}^{2} H\right)^{1 / 3}$, where $d_{0}$ is the minimum penetration depth for $h=0$. However, as in ref. 7, for steel spheres and glass beads ranging from 45 to $425 \mu \mathrm{m}$, the penetration depth, $d$, is also well approximated by $d_{0}+\alpha\left|v_{0}\right|$. Though the experimental systems of refs 6-10 are all different, and were interpreted by four different force laws, they are actually all consistent with the data reported here. This suggests that a single, unified, force law underlies all of the observations.

Tsimring and Volfson ${ }^{14}$ proposed that the total force on the projectile is

$$
\Sigma F=-m g+F(z)+m v^{2} / d_{1},
$$

equal to the sum of gravity plus Coulomb friction plus inertial drag. This is similar to the Poncelet force law, $F_{0}+c v^{2}$, used in high-speed ballistics ${ }^{11,12}$, but accounts for the depth-dependence of Coulomb friction in granular media. Tsimring and Volfson argued that the form of $F(z)$ should vary from quadratic to constant owing to the shapes of the projectile and of the growing crater excavated by its motion. They also showed how 


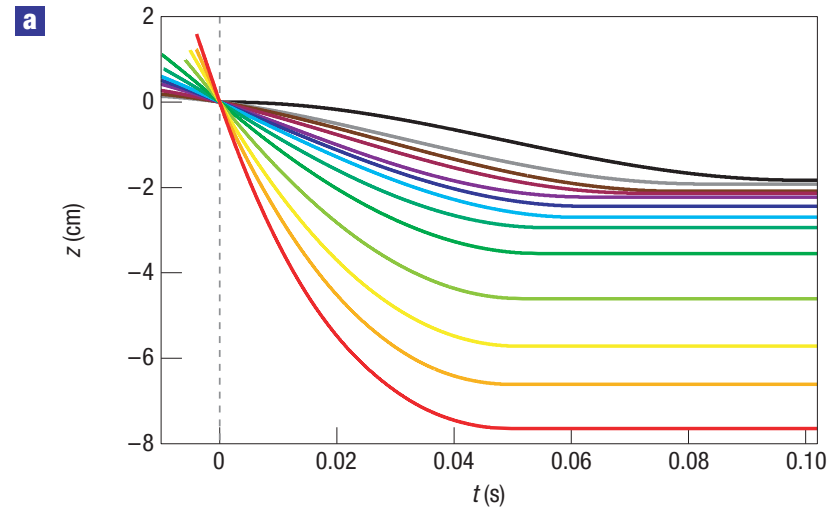

b

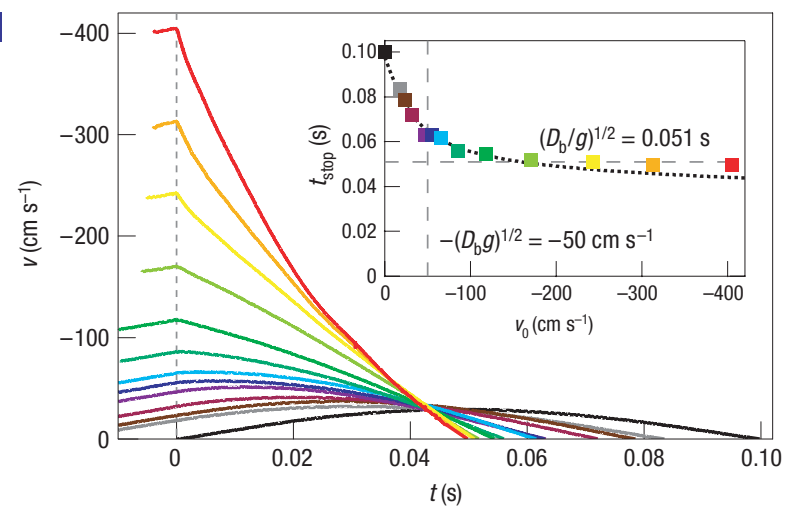

G

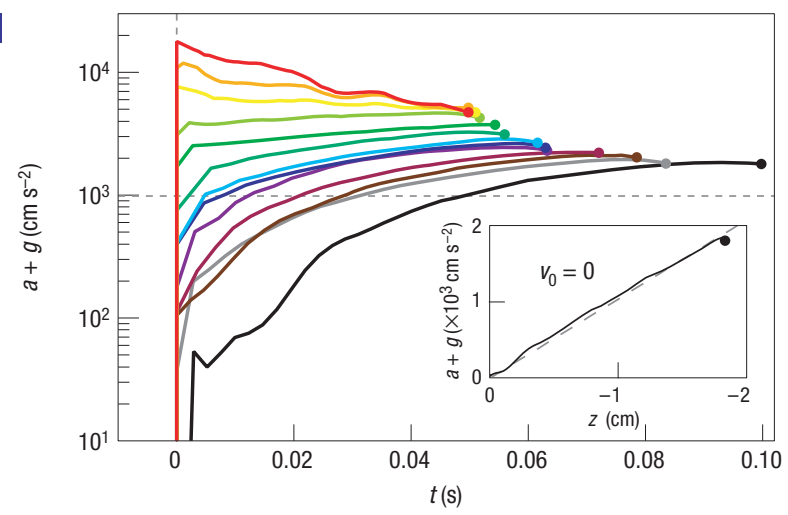

Figure 1 Kinematics of impact for a steel sphere dropped onto a bed of glass beads. a-c, Projectile depth, $z(t)$ (a); projectile speed, $v(t)$ (b); and net acceleration, $a(t)+g(\mathbf{c})$ versus time, $t$. The origin is defined by initial contact between the projectile and the medium, and position is measured upwards opposite to gravity. The curves are colour coded according to initial impact speed. Inset to $\mathbf{b}$ : Stopping time versus impact speed, along with the characteristic time and velocity scales set by projectile size and gravity. The dotted black curve shows the stopping time predicted by equation (1) with the parameters from Fig. 3. Inset to c: Net acceleration, $a+g$, versus depth for the case of zero initial impact speed, along with the expectation, $k|z|$, on the basis of Coulomb friction alone.

such a force law can approximately account for a depth scaling of $d=\left(d_{0}^{2} H\right)^{1 / 3}$.

The entire dynamics data set of Fig. 1 can be used to test the form of equation (1). Combining it with Newton's second law, the acceleration at a given fixed depth $z_{i}$ should

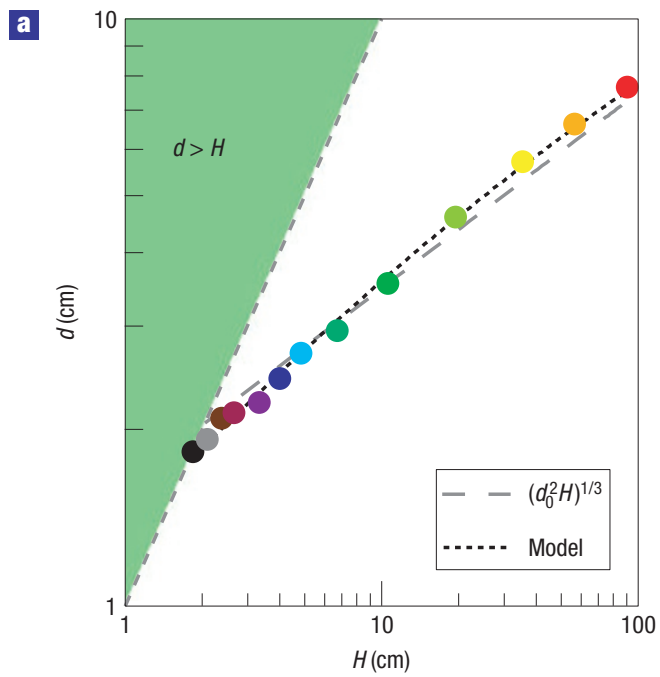

b

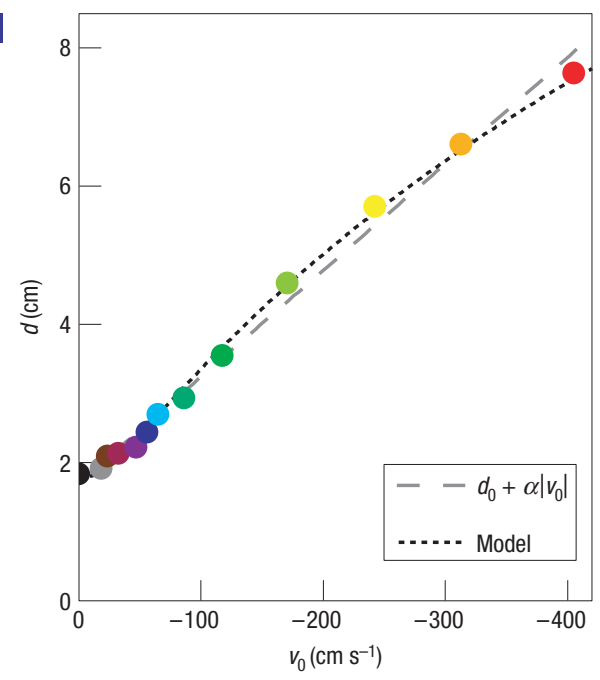

Figure 2 Scaling behaviour of the absolute final penetration depth, $\boldsymbol{d}$. a, Depth versus total drop distance, $H=h+d$, where $h$ is the free-fall distance. b, Depth versus initial impact speed, $v_{0}=-(2 g h)^{1 / 2}$. The symbols are colour coded according to the initial impact speed as in Fig. 1. The dashed grey lines show previously reported empirical scaling rules: a, $d=\left(d_{0}^{2} H\right)^{1 / 3}$, where $d_{0}$ is the fitting parameter ${ }^{6,13}$; and $\mathbf{b}, d=d_{0}+\alpha\left|v_{0}\right|$, where both $d_{0}$ and $\alpha$ are fitting parameters ${ }^{7}$. The dotted black curve shows the depth predicted by equation (1) with parameters from Fig. 3.

be quadratic in speed: $a+g=F\left(z_{i}\right) / m+v^{2} / d_{1}$. For each drop height, we therefore examine the acceleration and the speed when the projectile passes through five different fixed depths, $z_{i}=\{0,-1,-2,-3,-4\} \pm 0.1 \mathrm{~cm}$. The acceleration values are shown versus speed in Fig. 3a, where each point represents a different drop height and where the five colours represent the five fixed depths. The results are quadratic in speed and furthermore, crucially, have the same proportionality factor $d_{1}=8.7 \pm 0.7 \mathrm{~cm}$ for all five depths. This is demonstrated by the dotted curves in the main plot, which become parallel lines when plotted versus speed squared in the inset. The good agreement shows that the projectile experiences a force $m v^{2} / d_{1}$ that is independent of depth. This result can be expressed as $0.8 \rho_{\mathrm{g}} D_{\mathrm{b}}^{2} v^{2}$, and hence can be interpreted as an inertial force required for the projectile to mobilize a volume $D_{\mathrm{b}}^{3}$ of granular media with density $\rho_{\mathrm{g}}$. 
a

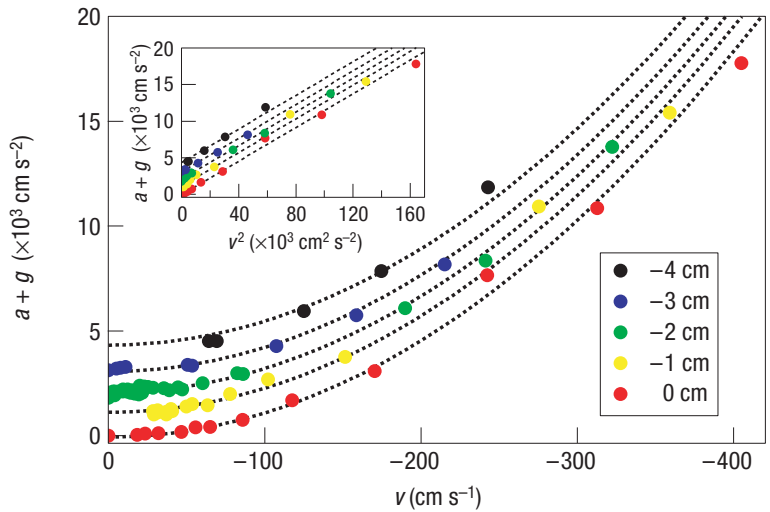

b

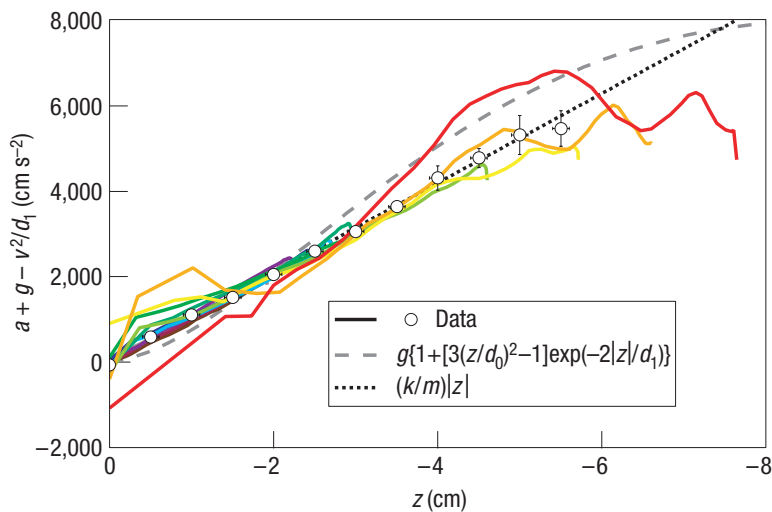

Figure 3 Depth and velocity dependence of the stopping force.

a, Net acceleration, $a+g$, versus velocity, at five specific fixed depths, $z_{i}$. The coloured points represent data. The dotted black curves represent fits to $a+g=F\left(z_{i}\right) / m+v^{2} / d_{1}$, where $d_{1}$ is set to $8.7 \mathrm{~cm}$ and $F\left(z_{i}\right) / m$ is adjusted for each depth. The inset shows the same plot but versus $v^{2}$, to demonstrate the quadratic dependence on speed and the constancy of $d_{1}$. $\mathbf{b}$, Net acceleration, $a+g-v^{2} / d_{1}$, versus depth, $z$; according to equation (1), this should collapse all data to the friction force $F(z) / m$. The coloured curves represent data, and are colour coded according to the initial impact speed as in Fig. 1. The open symbols represent fitting results for the intercept, $F\left(z_{i}\right) / m$ at $v=0$, for several fixed heights including those in $\mathbf{a}$; the vertical error bars represent fitting uncertainty and the horizontal error bars represent the range of heights included in the fits. The dashed and dotted curves represent candidate forms for $F(z) / m$ versus $z$, as labelled.

The form of the Coulomb friction-like term may now be examined using the value of $d_{1}$ deduced above. As equation (1) gives $F(z) / m=a+g-v^{2} / d_{1}$, we evaluate the right-hand side and plot the results versus depth in Fig. $3 b$. There the curves represent data for different drop heights; the open symbols represent extrapolation to $v=0$ of $a+g$ data at fixed depth for many drop heights, from Fig. 3a. This produces a good collapse of our entire data set, for all drop heights and for all times. It shows that the projectile experiences a force $F(z)$ that is independent of speed and that increases with depth. The form of this force is similar to $F(z) /(m g)=1+\left[3\left(z / d_{0}\right)^{2}-1\right] \exp \left(-2|z| / d_{1}\right)$, shown in Fig. 3b as a grey dashed curve, which is constructed to give $d=\left(d_{0}^{2} H\right)^{1 / 3}$ exactly ${ }^{15}$. This varies from quadratic to linear, and saturates at great depths, just as argued by Tsimring and Volfson. However, the data are better fitted over most of the range to a simpler, linear form $F(z)=k|z|$ with $k / m=1,040 \pm 10 \mathrm{~s}^{-2}$, shown by the dotted line. This result can be expressed as $k=20 \mu \rho_{\mathrm{g}} g D_{\mathrm{b}}^{2}$, which is larger than expected for ordinary Coulomb friction and observed previously for objects pushed horizontally in a granular medium ${ }^{16}$.

Altogether we have shown that a force law, $\Sigma F=-m g+k|z|+m v^{2} / d_{1}$, can account for the salient features of existing granular impact data. Here, the high quality and range of the dynamics data are sufficient to allow the individual depthand velocity-dependent terms to be isolated and demonstrated. The order of magnitude of the latter force is $\rho_{\mathrm{g}} D_{\mathrm{b}}^{2} v^{2}$, set by grain density and ball area. The order of magnitude of the former force is larger than $\mu \rho_{\mathrm{g}} g D_{\mathrm{b}}^{2}|z|$, set by Coulomb friction. Although the detailed scaling has yet to be checked, the order-of-magnitude consistency of these expressions reveal that the characteristic length scale is given by the diameter of the projectile, $L_{\mathrm{c}}=D_{\mathrm{b}}$. This explains why the penetration depth is within an order of magnitude of the ball diameter for a wide range of impact speeds. As gravity is the only system parameter whose units contain time, the characteristic time and velocity scales must therefore be $T_{\mathrm{c}}=\left(D_{\mathrm{b}} / g\right)^{1 / 2}$ and $V_{\mathrm{c}}=\left(D_{\mathrm{b}} g\right)^{1 / 2}$. Indeed, these explain the typical stopping time and the velocity beyond which the stopping time is constant, seen in the inset of Fig. 1b. In addition, finally, the force law explains the acceleration discontinuity at stoppage, seen in Fig. 1c, as $\Delta a=-g+(k / m) d$. The only puzzles that remain are the linear form of the Coulomb friction term plus the precise values of $k$ and $d_{1}$, as well as their scaling with system properties. In addition to accounting for the full behaviour of the projectile, the fundamental forces demonstrated here to act between the projectile and the medium must also govern the injection of energy needed to explain spectacular features in the medium such as crater morphologies ${ }^{4,5}$ and jet splash heights ${ }^{17-20}$.

\section{METHODS}

Spherical glass beads (diameter range $250-350 \mu \mathrm{m}$ ) are used as a dry non-cohesive granular medium with density $\rho_{\mathrm{g}}=1.52 \mathrm{~g} \mathrm{~cm}^{-3}$, draining angle of repose $\theta_{r}=24^{\circ}$ and friction coefficient $\mu=\tan \left(\theta_{r}\right)=0.45$. A clear plexiglass tube (outer diameter: 8 inches; inner diameter: 7.5 inches; height: 12 inches) is set on a sieve (US sieve size 45-60). The same windbox used in previous experiments ${ }^{13}$ is attached under this container. The glass beads are poured into the container to a depth of about $20 \mathrm{~cm}$. The medium is fluidized, and gradually de-fluidized, by a uniform upflow of $\mathrm{N}_{2}$ gas before each impact to ensure a homogeneous medium with a flat surface. The volume fraction occupied by the beads is $0.590 \pm 0.004$ after fluidization ${ }^{21}$. A steel sphere of diameter $D_{\mathrm{b}}=2.54 \mathrm{~cm}$ is used as a projectile. An acrylic transparent square rod (length: 8 inches; square cross-section: $1 / 8 \times 1 / 8$ inches) is glued vertically on top of the sphere. A horizontally striped transparent sheet is affixed to one side of the rod. The width and space of the stripes are $0.2 \mathrm{~mm}$. A small metal tip is glued to the top of the rod. The total mass of the projectile plus rod is $m=69.2 \mathrm{~g}$, giving the ball an effective density of $\rho_{\mathrm{b}}=8.07 \mathrm{~g} \mathrm{~cm}^{-3}$. The projectile is held by an electromagnet, which is turned off to commence free-fall of the projectile. A line-scan CCD camera (1,024 pixels, 8 bits deep, $50 \mathrm{kHz}$ frame rate) is placed to capture light transmitted through the striped pattern. Images are also acquired before and after each impact for calibration of length scale.

To analyse video data, the velocity is first deduced from the frame rate and the location of the peak in the cross correlation of two successive images of the striped pattern. Next, the impact time and speed are identified from fits of raw $v(\tau)$ data to $\left[v_{0}-g\left(\tau-\tau_{0}\right)\right] H\left(\tau-\tau_{0}\right)+$ $\left[v_{0}+a_{0}\left(\tau-\tau_{0}\right)+j_{0}\left(\tau-\tau_{0}\right)^{2} / 2\right] H\left(\tau_{0}-\tau\right)$, where the fitting parameters are $\left\{v_{0}, a_{0}, j_{0}, \tau_{0}\right\}$ and $H(x)$ is the Heaviside function. The stop time and acceleration discontinuity are identified by fits to $v(t)=\Delta a\left(t-t_{\text {stop }}\right)$ over a small window before $t_{\text {stop }}$, where $t=\tau-\tau_{0}$. Position is then deduced from $z(t)=\int_{0}^{t} v\left(t^{\prime}\right) \mathrm{d} t^{\prime}$. The results are consistent with, but more accurate than, the values of $h=v_{0}^{2} /(2 g)$ and $d=-z(\infty)$ measured directly by a microtelescope mounted to a height gauge. Finally, acceleration is deduced from fits of the velocity data to line segments, where the fitting windows are adjusted so that the acceleration uncertainty is smaller than the larger of $0.5 \%$ or $0.005 \mathrm{~g}$. An example of raw video data and analysis is given in the Supplementary Information, along with a discussion of possible effects due to atmospheric air pressure. 


\section{Received 7 December 2006; accepted 5 March 2007; published 1 April 2007.}

\section{References}

1. Amato, J. C. \& Williams, R. E. Crater formation in the laboratory: An introductory experiment in error analysis. Am. J. Phys. 66, 141-143 (1998).

2. Walsh, A. M., Holloway, K. E., Habdas, P. \& de Bruyn, J. R. Morphology and scaling of impact craters in granular media. Phys. Rev. Lett. 91, 104301 (2003).

3. Daniels, K. E., Coppock, J. E. \& Behringer, R. P. Dynamics of meteor impacts. Chaos 14, S4 (2004).

4. Zheng, X.-J., Wang, Z.-T. \& Qiu, Z.-G. Impact craters in loose granular media. Eur. Phys. J. E 13, 321-324 (2004)

5. Boudet, J. F., Amarouchene, Y. \& Kellay, H. Dynamics of impact cratering in shallow sand layers. Phys. Rev. Lett. 96, 158001 (2006).

6. Uehara, J. S., Ambroso, M. A., Ojha, R. P. \& Durian, D. J. Low-speed impact craters in loose granular media. Phys. Rev. Lett. 90, 194301 (2003).

7. de Bruyn, J. R. \& Walsh, A. M. Penetration of spheres into loose granular media. Can. J. Phys. 82, 439-446 (2004)

8. Ciamarra, M. P. et al. Dynamics of drag and force distributions for projectile impact in a granular medium. Phys. Rev. Lett. 92, 194301 (2004).

9. Lohse, D., Rauhe, R., Bergmann, R. \& van der Meer, D. Creating a dry variety of quicksand. Nature 432, 689-690 (2004)

10. Hou, M., Peng, Z., Liu, R., Lu, K. \& Chan, C. K. Dynamics of a projectile penetrating in granular systems. Phys. Rev. E 72, 062301 (2005).

11. Backman, M. E. \& Goldsmith, W. Mechanics of penetration of projectiles into targets. Int. J. Eng. Sci. 16, 1-99 (1978).

12. Zukas, J. A. (ed.) High Velocity Impact Dynamics (Wiley, New York, 1990).

13. Ambroso, M. A., Santore, C. R., Abate, A. R. \& Durian, D. J. Penetration depth for shallow impact cratering. Phys. Rev. E 71, 051305 (2005)
14. Tsimring, L. S. \& Volfson, D. in Powders and Grains 2005 (eds Garcia-Rojo, R., Herrmann, H. J. \& McNamara, S.) 1215-1223 (A.A. Balkema, Rotterdam, 2005).

15. Ambroso, M. A., Kamien, R. D. \& Durian, D. J. Dynamics of shallow impact cratering. Phys. Rev. E 72, 041305 (2005).

16. Albert, I. et al. Granular drag on a discrete object: Shape effects on jamming. Phys. Rev. E 64, 061303 (2001).

17. Cook, M. A. \& Mortensen, K. S. Impact cratering in granular materials. J. Appl. Phys. 38, 5125-5128 (1967).

18. Thoroddsen, S. T. \& Shen, A. Q. Granular jets. Phys. Fluids 13, 4-6 (2001)

19. Lohse, D. et al. Impact on soft sand: Void collapse and jet formation. Phys. Rev. Lett. 93 , 198003 (2004).

20. Royer, J. R. et al. Formation of granular jets observed by high-speed X-ray radiography. Nature Phys. 1, 164-167 (2005).

21. Ojha, R., Menon, N. \& Durian, D. J. Hysteresis and packing in gas-fluidized beds. Phys. Rev. E 62, $4442-4445$ (2000).

\section{Acknowledgements}

This work was supported by the National Science Foundation (D.J.D.) and the Japan Society for the Promotion of Science Postdoctoral Fellowships for Research Abroad (H.K.)

Correspondence and requests for materials should be addressed to D.J.D.

Supplementary Information accompanies this paper on www.nature.com/naturephysics.

\section{Competing financial interests}

The authors declare no competing financial interests.

Reprints and permission information is available online at http://npg.nature.com/reprintsandpermissions/ 